\section{Identification des progéniteurs de l'épithélium intestinal fœtal}

Marie-Isabelle Garcia, Gilbert Vassart
Institut de recherche interdisciplinaire en biologie humaine et moléculaire (IRIBHM), Faculté de médecine, Université libre de Bruxelles, route de Lennik 808, 1070 Bruxelles, Belgique.

mgarcia@ulb.ac.be

gvassart@ulb.ac.be

sous forme d'organoïdes, aussi décrits comme des «mini-intestins adultes 》 [6]. Les intestins de souris ont été prélevés à différents stades du développement fœtal et postnatal, puis cultivés ex vivo [7]. Nous avons constaté que la culture d'intestins à $\varepsilon 14$ génère, non pas des organoïdes, mais des sphéroïdes constitués de cellules épithéliales polarisées monostratifiées d'origine intestinale, capables de renouvellement à long terme. La proportion des sphéroïdes produits en culture décroît au cours du développement, ces derniers étant progressivement remplacés par des organoïdes, tels qu'on les obtient par la culture de cellules souches adultes. Ceci indique que les cellules générant les sphéroïdes sont le reflet d'un stade particulier du développement de l'épithélium intestinal fœetal.

\section{Un transcriptome différent de celui des CBC}

Le profil d'expression génique de ces sphéroïdes fœtaux diffère de celui des organoïdes par l'absence d'expression de marqueurs de différenciation des lignées cellulaires de l'épithélium adulte et par des niveaux faibles, voire indétectables, des marqueurs de $C B C$, tels que le récepteur Lgr5. L'analyse de cultures d'intestins provenant de souris invalidées pour le récepteur Lgr5 a montré que ce marqueur n'est pas requis pour la croissance des sphéroïdes, au contraire du récepteur paralogue Lgr4 qui, lui, est apparu essentiel pour leur génération [7]. Bien que radicalement différents des CBC, les sphéroïdes fœtaux sont caractérisés par l'expression de gènes décrits comme marqueurs de cellules 


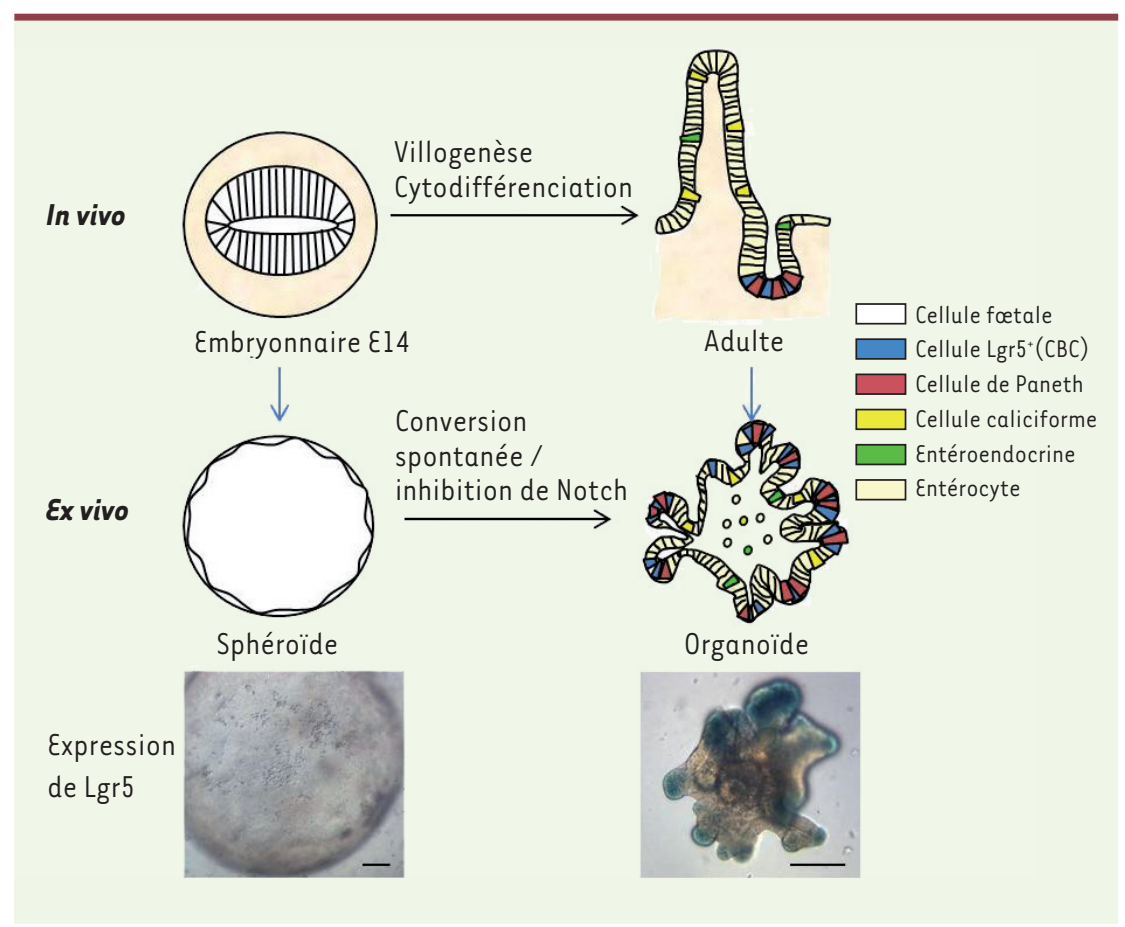

Figure 1. Représentation schématique de l'épithélium intestinal murin aux stades embryonnaire (ع14) et adulte. Les cellules progénitrices fœtales et souches adultes $\operatorname{Lgr} 5^{+}$génèrent respectivement des sphéroïdes et des organoïdes lorsqu'elles sont cultivées ex vivo. L'expression de Lgr5, marqueur de cellules souches adultes, y est visualisée par mesure de l'activité $\beta$-galactosidase (cellules bleues) à partir de la lignée de souris transgénique Lgr5-LacZ. Échelle : $200 \mu \mathrm{m}$.

souches/progénitrices ou impliqués dans la régénération cellulaire dans certains tissus. La pertinence in vivo des marqueurs de sphéroïdes (cultivés ex vivo) a été ensuite confirmée en analysant leur expression sur de l'épithélium intestinal d'embryons de souris. Parmi ces marqueurs figurent le tumorassociated calcium signal transducer 2 (Trop2/Tacstd2), proposé comme marqueur diagnostique de cancer colorectal, et la connexine 43 , aussi nommée gap junction protein 1 ( $\mathrm{C} \times 43 / \mathrm{Gjal})$ car impliquée dans les communications intercellulaires $[8,9]$. Au stade précédant la phase de villogenèse $(\varepsilon 14)$, ces deux molécules apparaissent fortement exprimées dans l'épithélium, tandis que leur expression disparaît progressivement après le début de la cytodifférenciation [7]. Des expériences complémentaires de traçage cellulaire ont été réalisées à l'aide de souris transgéniques Cnx43-CreER/Rosa26R exprimant impliquées dans les villogenèses fœtale et adulte, la conversion de sphéroïdes fœtaux en organoïdes adultes a été recherchée ex vivo. Une conversion spontanée a pu être constatée, conduisant à l'apparition de CBC dans les organoïdes néoformés, mais elle est peu active [7]. Ce taux de conversion augmente si les cultures sont traitées avec des agents modulant négativement la cascade de signalisation Notch, voie bien connue pour contrôler la différenciation des cellules souches intestinales [7]. Bien que les mécanismes moléculaires impliqués dans la transition de sphéroïde à organoïde ne soient pas encore élucidés, ces données indiquent toutefois que les cellules fœtales ont la capacité intrinsèque de se convertir en cellules souches adultes et suggèrent l'existence d'un processus similaire in vivo.

Dans un article paru simultanément au nôtre, Fordham et al. ont aussi rapporté l'existence de cellules fœtales murines et humaines (au stage gestationnel de 10 semaines) de l'épithélium intestinal capables de générer des sphéroïdes ex vivo [10]. Dans une expérience destinée à établir la preuve de concept, les cellules de sphéroïdes fœtaux murins ont pu être utilisées comme source de greffe dans un modèle de colite induite dans des souris adultes immunodéficientes; elles se montrent capables de générer des cellules différenciées dans le tissu hôte [10].

L'identification de cellules progénitrices à l'origine de l'épithélium fotal intestinal et en amont des cellules souches adultes laisse penser que des types de cellules similaires pourraient exister dans d'autres organes du tube digestif embryonnaire. L'isolement et la caractérisation de ces populations cellulaires feront certainement l'objet de recherches futures. $\diamond$

identification of progenitors of the foetal intestinal epithelium

\section{LIENS D'INTÉRÊT}

\section{Conversion des sphéroïdes en organoïdes}

Afin d'établir s'il existe un lien possible entre ces deux types de cellules
Les auteurs déclarent n'avoir aucun lien d'intérêt concernant les données publiées dans cet article. 


\section{RÉFÉRENCES}

1. Joubert D, Hollande F, Jay P, Legraverend C. Les cellules souches intestinales, 30 ans d'une histoire exemplaire. Med Sci (Paris) 2009; 25 : 441-4.

2. Romagnolo B. Une relation Paneth entre cellules souches et niche intestinale. Med Sci (Paris) $2012 ; 28$ : 1058-60

3. Barker N, van $\varepsilon s J H$, Kuipers J, et al. Identification of stem cells in small intestine and colon by marker gene Lgr5. Nature 2007 ; 449 : 1003-7.

4. Buczacki SJ, Zecchini HI, Nicholson AM, et al. Intestinal label-retaining cells are secretory precursors expressing Lgr5. Nature $2013 ; 495: 65-9$.
5. Spence JR, Lauf R, Shroyer NF. Vertebrate intestinal endoderm development. Dev Dyn 2011 ; 240 : 501-20.

6. Sato T, Vries RG, Snippert HJ, et al. Single Lgr5 stem cells build crypt-villus structures in vitro without a mesenchymal niche. Nature 2009 ; 459 : 262-5.

7. Mustata RC, Vasile G, Fernandez-Vallone V, et al. Identification of Lgr5-independent spheroidgenerating progenitors of the mouse fetal intestinal epithelium. Cell Rep $2013 ; 5: 421-32$.

8. Trerotola M, Cantanelli P, Guerra $\varepsilon$, et al. Upregulation of Trop-2 quantitatively stimulates human cancer growth. Oncogene $2013 ; 32: 222-33$
9. Kar R, Batra N, Riquelme MA, Jiang JX. Biological role of connexin intercellular channels and hemichannels. Arch Biochem Biophys 2012 ; 524 : 2-15.

10. Fordham RP, Yui S, Hannan NR. Transplantation of expanded fetal intestinal progenitors contributes to colon regeneration after injury. Cell Stem Cell 2013; $13: 734-44$.

\section{NOUVELLE}

\section{Les tumeurs dormantes prises au piège de la récidive}

Nicolas Boisgerault, Richard G. Vile
Mayo Clinic, Department of Molecular Medicine, 200 First Street SW, Guggenheim 18, Rochester, MN, 55905, États-Unis. boisgerault.nicolas@mayo.edu vile.richard@mayo.edu
$>$ Le suivi des tumeurs dormantes et la détection précoce des récidives tumorales constituent des défis majeurs en cancérologie. La phase de dormance est la période durant laquelle certaines cellules malignes ayant échappé au traitement de la tumeur primaire demeurent au site tumoral à l'état latent [1]. Chez les patients, cette période peut s'étendre de plusieurs mois à plusieurs années et précède souvent l'émergence de récidives agressives, conséquences de profondes modifications phénotypiques acquises sous la pression de sélection du microenvironnement, des cellules immunitaires et des traitements antitumoraux. Les mécanismes qui président à la réactivation des tumeurs dormantes sont largement méconnus et il demeure extrêmement difficile de prédire l'émergence de récidives ou leur sensibilité aux traitements disponibles.

Afin d'étudier les mécanismes tumoraux impliqués dans ces différents stades de la progression tumorale, nous avons récemment développé différents modèles murins de mélanome et d'adénocarcinome de la prostate. Dans ces modèles, les cellules tumorales sont traitées de façon sous-optimale par immunothéra- pie, virothérapie ou chimiothérapie afin de provoquer une régression significative de la tumeur primaire tout en permettant la survie de quelques cellules à l'état latent, puis l'émergence de récidives. Dans un travail précédent, nous avions ainsi identifié une sous-population de cellules tumorales, responsables de l'initiation du processus de récidive, qui présentent des propriétés plastiques leur permettant d'ajuster leur phénotype en fonction des pressions de l'environnement [2]. Ces cellules se caractérisent notamment par une surexpression transitoire de la topoisomérase-ll $\alpha$ qui peut être ciblée de façon spécifique par l'utilisation de doxorubicine afin de prévenir l'émergence de récidives.

\section{Détection précoce des récidives tumorales}

Notre dernière étude visait à caractériser plus précisément les tumeurs dormantes et à déterminer si les récidives pouvaient être détectées à un stade précoce et avant tout autre signe clinique [3]. Des travaux précédents [4], en particulier sur la transition angiogénique [5, 6] $(\rightarrow)$, suggéraient

$(\rightarrow)$ Voir $m / s n^{\circ} 12$, décembre 2012, page 1069 notamment que certaines cytokines intervenaient dans ces mécanismes. Nous avons effectivement découvert que l'apparition de récidives chez l'animal était précédée de la sécrétion transitoire de vascular endothelial growth factor (Vegf) et d'interleukine-6 (IL-6), qui pouvaient être détectés de façon systémique dans le sérum. En combinant l'utilisation d'un plasmide contenant le gène de la luciférase sous le contrôle du promoteur du gène Vegf et l'imagerie de bioluminescence in vivo, l'apparition de récidives pouvait ainsi être anticipée jusqu'à douze jours avant que la tumeur ne devienne décelable par les techniques classiques.

Les sécrétions de Vegf et d'IL-6 sont liées à l'activation d'une réponse immunitaire innée qui reconnaît la reprise soudaine de la prolifération tumorale. Afin d'échapper à cette réponse immune puis d'évoluer vers une récidive de taille plus importante, les cellules malignes doivent alors modifier leur phénotype en profondeur. Nous avons ainsi montré qu'au cours du processus de récidive, ces cellules acquièrent une forte insensibilité aux effecteurs innés, tout en étant reconnues plus efficacement par 